\title{
Abordaje anterolateral extendido y fijación con placa rim en fragmentos posterolaterales en fracturas de platillo tibial
}

\section{Extended Anterolateral Approach and Rim Plate Fixation for Posterolateral Fragments in Tibial Plateau Fractures}

\author{
Rodrigo Guiloff ${ }^{1,2}$ Magaly Iñiguez ${ }^{1,3}$ Alfredo Sandoval ${ }^{1}$ Franciso Figueroa ${ }^{1,2}$ Tomás Prado ${ }^{1}$ \\ Sebastián Cabrera ${ }^{1}$ Oscar Colmenares ${ }^{1}$ Eduardo Carrasco ${ }^{1,4}$
}

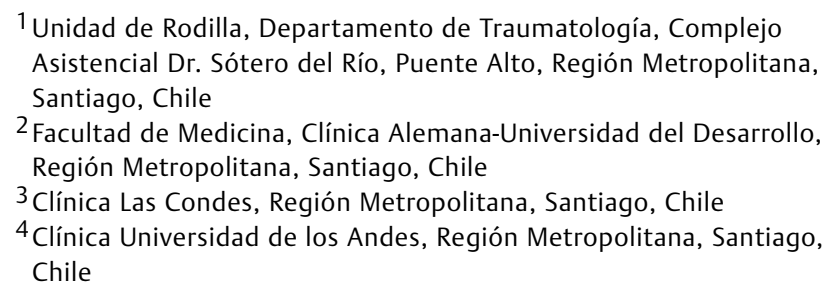

Dirección de correspondencia Rodrigo Guiloff, Clínica Alemana de Santiago SA, avenida Vitacura 5.951, Vitacura, Región Metropolitana, Santiago, Chile (e-mail: rguiloff@gmail.com).

Rev Chil Ortop Traumatol 2021;62:39-45.

\section{Resumen \\ Palabras claves \\ - abordaje \\ - fractura \\ - fragmento posterolateral \\ - placa rim \\ - platillo tibial}

Los fragmentos posterolaterales (FPLs) en fracturas de platillo tibial son frecuentes, pero difíciles de reducir y estabilizar. Actualmente existe controversia sobre cómo deben ser abordados; si bien un abordaje anterolateral es más seguro, este puede ser insuficiente para lograr una correcta reducción. Los abordajes posterolaterales y posteriores ofrecen una visión directa y permiten una fijación con ventaja biomecánica; sin embargo, son más demandantes y exponen al paciente a una mayor morbilidad. A continuación, se presenta una nota técnica sobre la reducción y fijación de FPLs con una placa rim por medio de un abordaje anterolateral extendido a través del espacio paraligamento colateral lateral.

Posterolateral fragments (PLFs) are commonly seen in tibial plateau fractures, but their reduction and fixation are challenging. There is no consensus about the ideal approach to fix this particular fragment. Even though an anterolateral approach is a safe option, it may impair a correct reduction. The posterolateral and posterior approaches offer direct visualization of the fragment, and enable a fixation with a biomechanical advantage; however, they are more demanding and expose the patient to a higher risk if morbidity. The following technical note describes the reduction and fixation of PLFs with a rim plate through an extended anterolateral approach using the paralateral collateral ligament space. recibido

21 de julio de 2020

aceptado

21 de enero de 2021
DOI https://doi.org/

$10.1055 / \mathrm{s}-0041-1728737$ ISSN 0716-4548.
(C) 2021. Sociedad Chilena de Ortopedia y Traumatologia. All rights reserved.

This is an open access article published by Thieme under the terms of the Creative Commons Attribution-NonDerivative-NonCommercial-License, permitting copying and reproduction so long as the original work is given appropriate credit. Contents may not be used for commercial purposes, or adapted, remixed, transformed or built upon. (https://creativecommons.org/ licenses/by-nc-nd/4.0/)

Thieme Revinter Publicações Ltda., Rua do Matoso 170, Rio de Janeiro, RJ, CEP 20270-135, Brazil 


\section{Introducción}

El concepto de fijación por columnas en fracturas de platillos tibiales fue expuesto por Luo y col. ${ }^{1}$ hace 10 años; sin embargo, la decisión de fijar los fragmentos posteriores a través de un abordaje posterior es aún controvertida. ${ }^{2}$ Según la clasificación actual de la AO Foundation/Orthopaedic Trauma Association (AO/OTA), modificada por Kfuri y Schatzker, ${ }^{3}$ el fragmento posterolateral (FPL) está ubicado en el platillo lateral posterior a la punta de la fíbula. ${ }^{3,4} \mathrm{Si}$ bien los FPLs son más frecuentes de lo que se creía (36\% a $80 \%$ ), ${ }^{5-7}$ se ha descrito una tasa de malreducción entre $50 \%$ y $70 \%{ }^{7}$

No existe consenso sobre cómo deben ser abordados los FPLs. ${ }^{8,9}$ El abordaje anterolateral es seguro y ampliamente conocido; sin embargo, ha demostrado ser insuficiente para lograr las correctas visualización, reducción y fijación de estos fragmentos. ${ }^{9}$ Los abordajes posterolaterales y posteriores ofrecen una visión directa y permiten una fijación con ventaja biomecánica; ${ }^{10,11}$ sin embargo, son técnicamente demandantes y exponen al paciente a una mayor morbilidad. ${ }^{12}$ Los abordajes posterolaterales y posteriores aumentan el riesgo de lesión del nervio peroneo común, la arteria tibial anterior, y el paquete ciático-poplíteo. ${ }^{13-15}$ Particularmente, los abordajes posteriores requieren operar al paciente en posición decúbito prono, y, en caso de existir un rasgo anterior a la línea media del platillo, requieren cambiar de posición al paciente durante el procedimiento, aumentando los tiempos quirúrgicos y, con ello, el riesgo de infección. ${ }^{16}$

A continuación, se ofrece una nota técnica sobre la reducción y fijación del FPL con placa rim a través de un abordaje anterolateral extendido, que utiliza el espacio paraligamento colateral lateral.

\section{Paciente y técnica quirúrgica}

\section{Paciente}

Se presenta el caso de un paciente de 75 años, hipertenso, quién, después de un atropello en la vía pública, sufrió una fractura de platillo tibial izquierdo clasificada como AO/OTA 43C3.1 $(\mathrm{t}, \mathrm{u})$, es decir, una fractura multifragmentaria del platillo tibial lateral con rasgos anterolateral y posterolateral (-Figura 1). El examen físico reveló un aumento de partes blandas y un alineamiento en valgo de la rodilla afectada.

El paciente fue debidamente informado antes y después de su cirugía, aceptó participar de esta investigación, y firmó un consentimiento informado.

\section{Abordaje anterolateral extendido}

En una mesa operatoria radiolúcida, el paciente es posicionado en decúbito supino, con la rodilla a operar en $20^{\circ}$ a $30^{\circ}$ de flexión y un alzador a nivel del glúteo ipsilateral. Se marcan los puntos anatómicos de referencia para el abordaje: tubérculo anterolateral de la tibial proximal (Gerdy), el reborde anterolateral de la tibia, la cabeza fibular, la línea articular lateral, el tubérculo anterior de la tibia proximal, y el tendón patelar. Se realiza una incisión curvilínea desde el tubérculo de Gerdy (TG) hacia la línea articular lateral, extendiéndose a proximal y a distal por sobre el reborde anterolateral de la tibia según necesidad. Se continúa con la elevación en colgajo del tejido subcutáneo hasta exponer la banda iliotibial (BIT) y su inserción en el TG. A continuación, se procede a incidir la BITy la fascia crural hacia distal, y, luego, a una elevación subperióstica del músculo tibial anterior. $\mathrm{Si}$ bien en el artículo original ${ }^{13}$ de este abordaje se indica que la BIT debe ser incidida en su línea media, los autores del presente estudio recomiendan evaluar caso a caso según el patrón de fractura y la asociación con más fragmentos. En este caso, se decidió incidir la fascia en su punto más anterior (dejando un remanente de $3 \mathrm{~mm}$ a $5 \mathrm{~mm}$ para su cierre posterior), para lograr una mejor exposición del fragmento anterolateral. Con el fragmento anterolateral expuesto, se agrega una artrotomía submeniscal, y el menisco lateral junto a la cápsula es elevado a través de suturas tipo encaje. Esto permite una adecuada visualización de la superficie articular y del menisco. Para aumentar la visualización, se recomienda realizar una maniobra de varo controlado.

A continuación, se procede a la extensión hacia la porción posterolateral de la tibia a través del espacio que Cho et al. ${ }^{17}$ acuñaron como parafibular colateral ligament space, o espacio paraligamento colateral lateral (-Figura 2). Al identificar el ligamento colateral lateral (LCL), se recomienda flectar la rodilla en $90^{\circ}$ para relajar el LCL y el nervio peroneo común. ${ }^{17}$ Con un retractor a través del espacio descrito, se separa el LCL hacia lateral y se procede a una disección del tejido cápsulo óseo del reborde del platillo lateral hasta exponer el FPL (- Figura 3A). Finalmente, el abordaje extendido permite separar el espacio para-LCL con un retractor de Hohmann y proceder a la reducción del fragmento, que puede realizarse en flexión o en extensión (- Figura 3B).

\section{Secuencia de reducción y utilización de placa rim}

En el caso descrito, se utilizó el rasgo y la diástasis anterolaterales para elevar aquellos fragmentos deprimidos en la conminución central descrita y el FPL. Por medio de agujas de Kirschner (K), se mantuvo la altura de los fragmentos, y, luego, se realizó la reducción del rasgo anterolateral. Se complementó la reducción con agujas en dirección anteroposterior con el objetivo de solidarizar ambos fragmentos, y se procedió a estabilizar con una placa bloqueada con ángulo variable de la tibia proximal lateral (Peri-loc VLP, Smith\&Nephew, Watford, Inglaterra, $\mathrm{RU})$, direccionando uno de los tornillos hacia el FPL. El FPL permaneció inestable, por lo que se extendió el abordaje anterolateral a través del espacio para-LCL para visualizar con detalle el fragmento, lograr una correcta reducción y, finalmente, una estabilización por medio de una placa rim. Para esta, se utilizó una placa de $2,7 \mathrm{~mm}$ bloqueada de forma trebolada, con ángulo variable, del sistema de medio/antepié (LCP, DePuy Synthes, Raynham, MA, EEUU).

La placa debe ser moldeada según la anatomía del paciente (-Figura 4). Es importante moldear la placa orificio tras orificio para conseguir una forma correcta, y se aconseja procurar que el extremo quede en contacto con el hueso. La - Figura 5 muestra el posicionamiento de la placa rim a través del abordaje anterolateral extendido, dejando el 


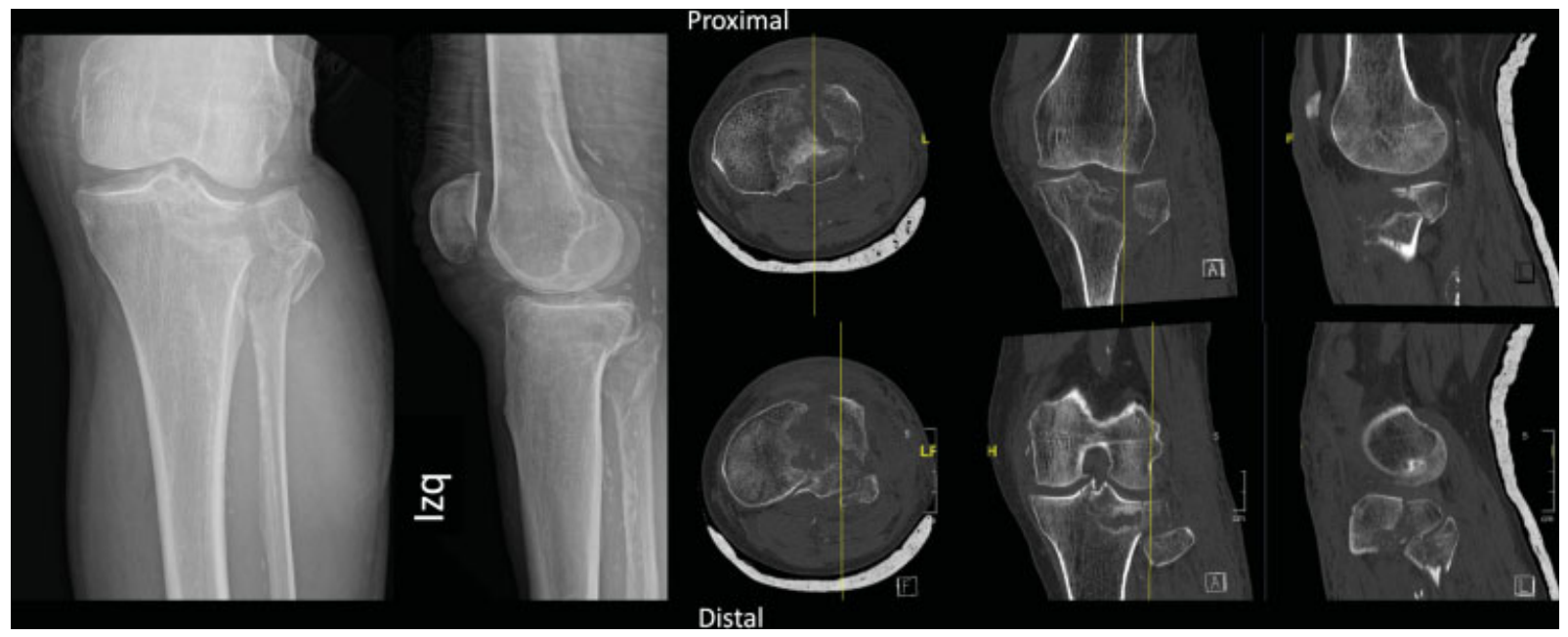

Fig. 1 Evaluación imagenológica preoperatoria. En la radiografía anteroposterior y lateral de rodilla izquierda, se evidencia una fractura de platillo tibial lateral, que muestra un ensanchamiento y una fractura de cabeza fibular asociada no desplazada. La tomografía computarizada confirma la presencia de un fragmento anterolateral y otro posterolateral, asociados a una conminución central. Según la clasificación actualizada de la AO/OTA (2018), corresponde a una AO/OTA 43C3.1(t,u). ${ }^{4}$

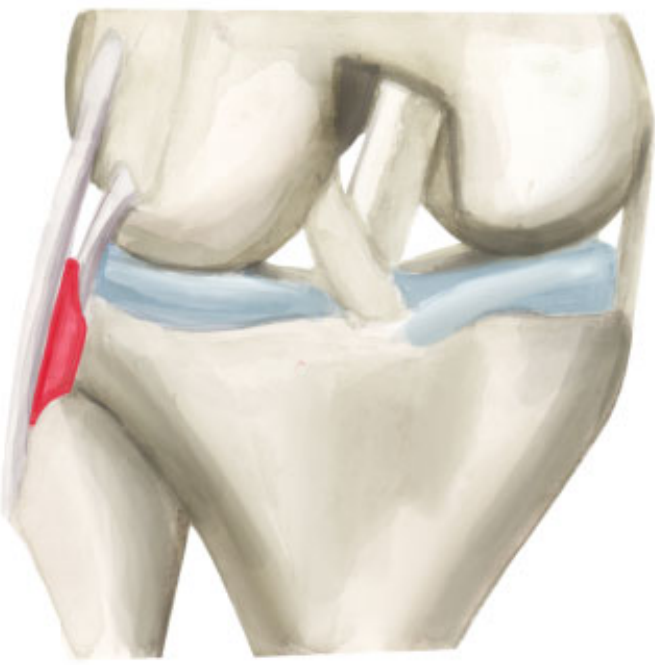

A

Fig. 2 Espacio paraligamento colateral lateral. (A) El espacio paraligamento colateral lateral (en rojo) se encuentra entre el ligamento colateral lateral y el reborde tibial lateral (visión anteroposterior). (B) La disección debe ser por debajo del vientre muscular del poplíteo $\left(^{*}\right)$ que discurre desde posteroinferior hacia anterosuperior (visión lateral). Redibujado de Cho et al. ${ }^{17}$

segmento trebolado hacia posterior, entregando contención al FPL que debe ser reducido. Para la fijación de la placa rim, se aconseja utilizar una pinza de reducción en el segmento posterior de la placa, y comenzar insertando un tornillo cortical en el primer orificio para lograr un correcto afrontamiento de la placa al hueso. Se introducen la mayor cantidad de tornillos posibles, procurando una dirección adecuada para mantener la reducción del FPL y la conminución central. Se puede aumentar la estabilización con tornillos en dirección anteroposterior. Se terminó la fijación colocando los tornillos de la placa lateral de tibia proximal (-Figuras 6 y 7 ).

\section{Rehabilitación}

Los autores del presente estudo recomiendan un protocolo de descarga de la extremidad operada con apoyo de bastones o andador por 6 a 8 semanas y, progresivamente, aumento de cargas, que será variable según el grado de conminución de la fractura. Ejercicios de rango articular pasivo-activo se inician en forma precoz para evitar rigidez, acompañados de isométricos de cuádriceps e isquiotibiales. Luego, según la sintomatología del paciente, se permiten ejercicios concéntricos en cadena cerrada inicialmente, y, cuando exista un adecuado control neuromuscular, se progresa a cadenas abiertas. 

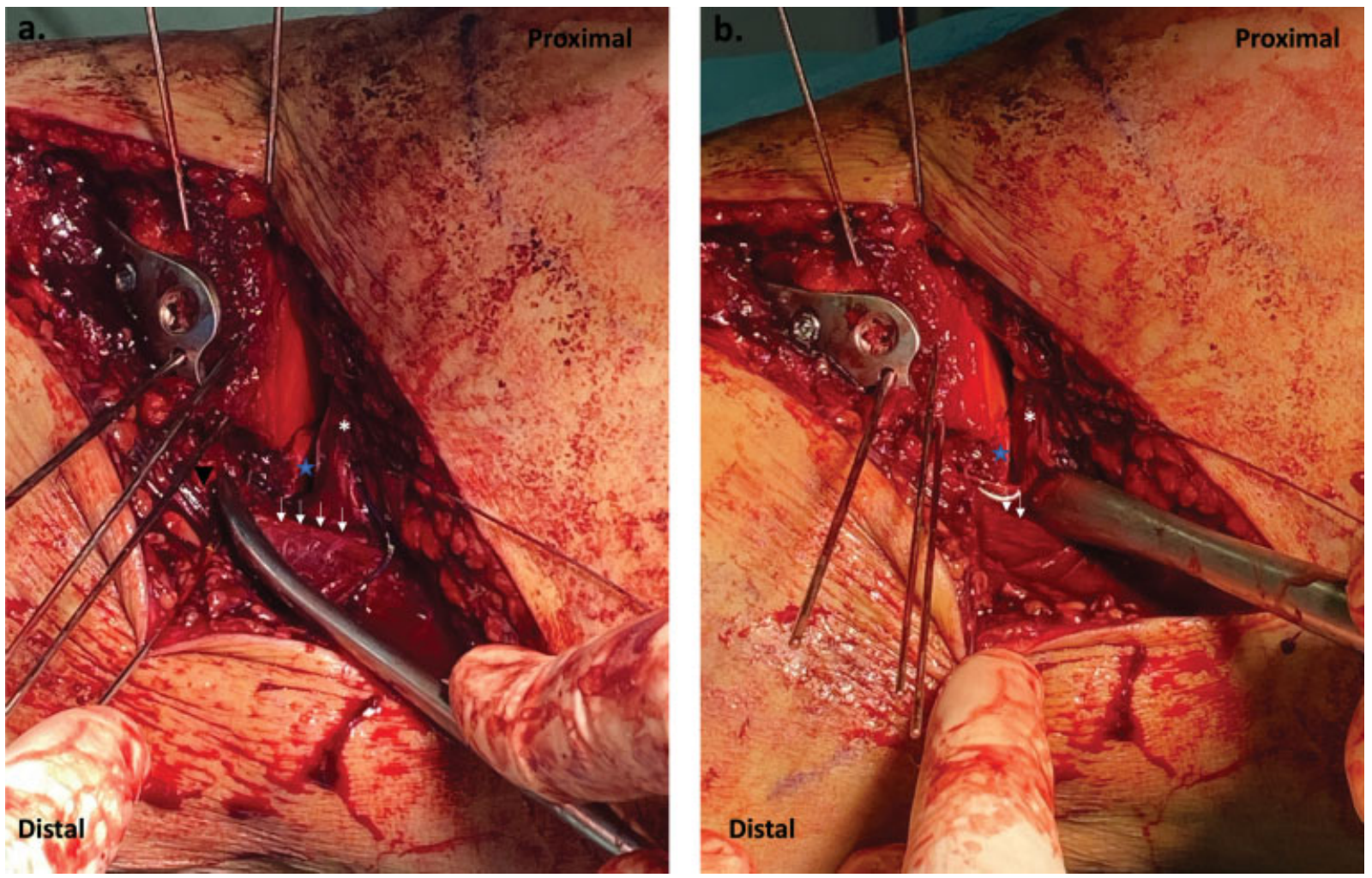

Fig. 3 Abordaje anterolateral extendido. (A) Se realizó una disección a través del espacio paraligamento colateral lateral (LCL, triángulo negro invertido), que, junto a la artrotomía submeniscal (asterisco blanco), permite una adecuada exposición del fragmento posterolateral (FPL) del platillo tibial (estrella azul). El margen anterior de la banda iliotibial por sobre el LCL se ilustra por medio de las flechas blancas. En este caso, se demuestra que, a pesar de un intento de reducción con agujas desde anterior a posterior a través del abordaje anterolateral clásico y el uso de un tornillo con ángulo variable a través de la placa dirigido hacia posterior, la reducción y estabilización del FPL fue insuficiente. (B) El abordaje anterolateral extendido permite colocar un reductor posterior al fragmento para lograr su reducción.
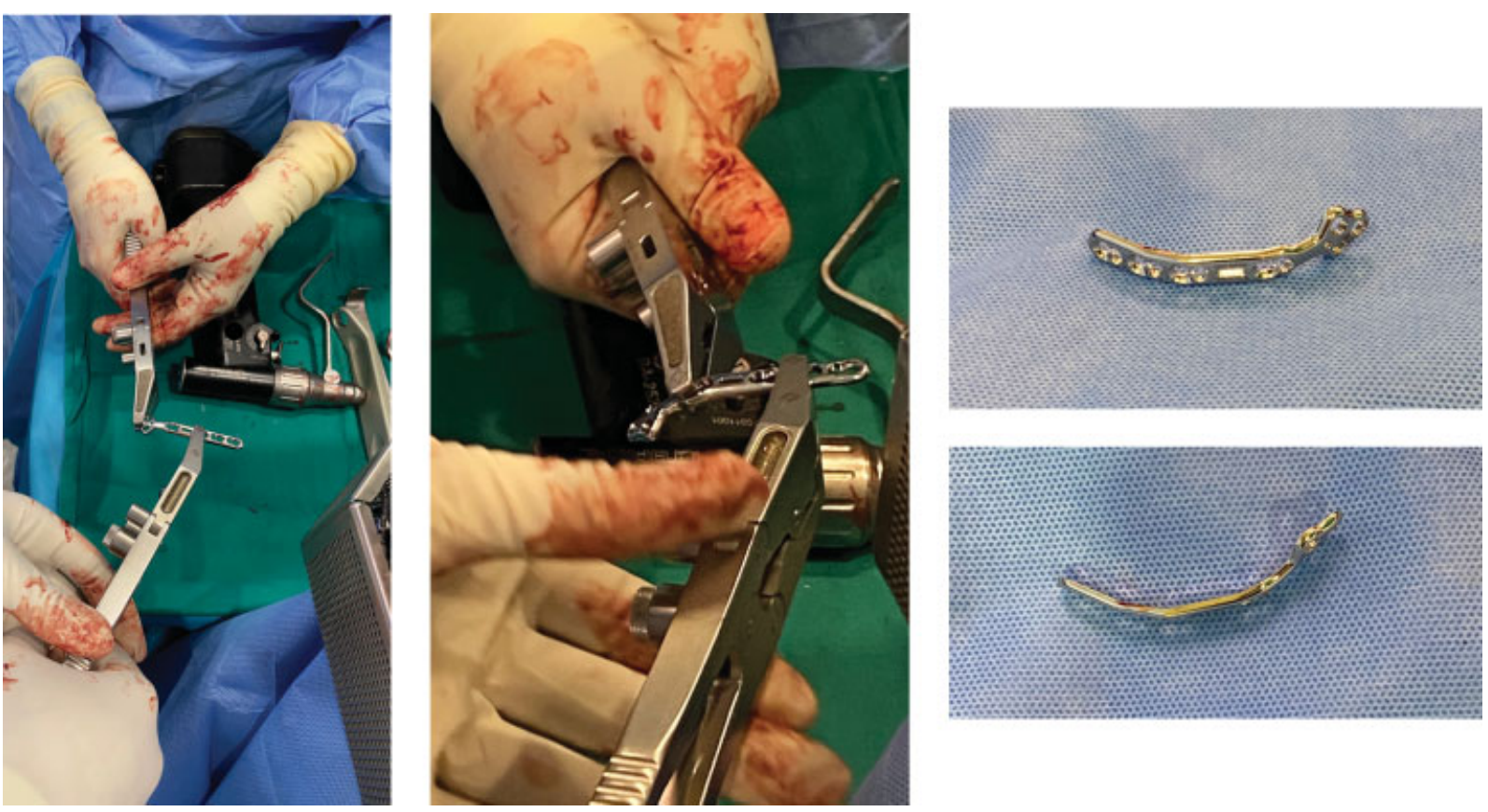

Fig. 4 Moldeado de placa rim: la placa debe ser moldeada en una mesa aparte según la anatomía del platillo del paciente. Se recomienda moldear la placa de forma parcelada (espacio tras espacio) para permitir una forma adecuada. Es importante utilizar el instrumental adecuado para moldear la placa; de lo contrario, los orificios podrían deformarse e impedir el bloqueo de tornillos. Se debe asegurar que se doble el extremo anterior para evitar prominencias. 

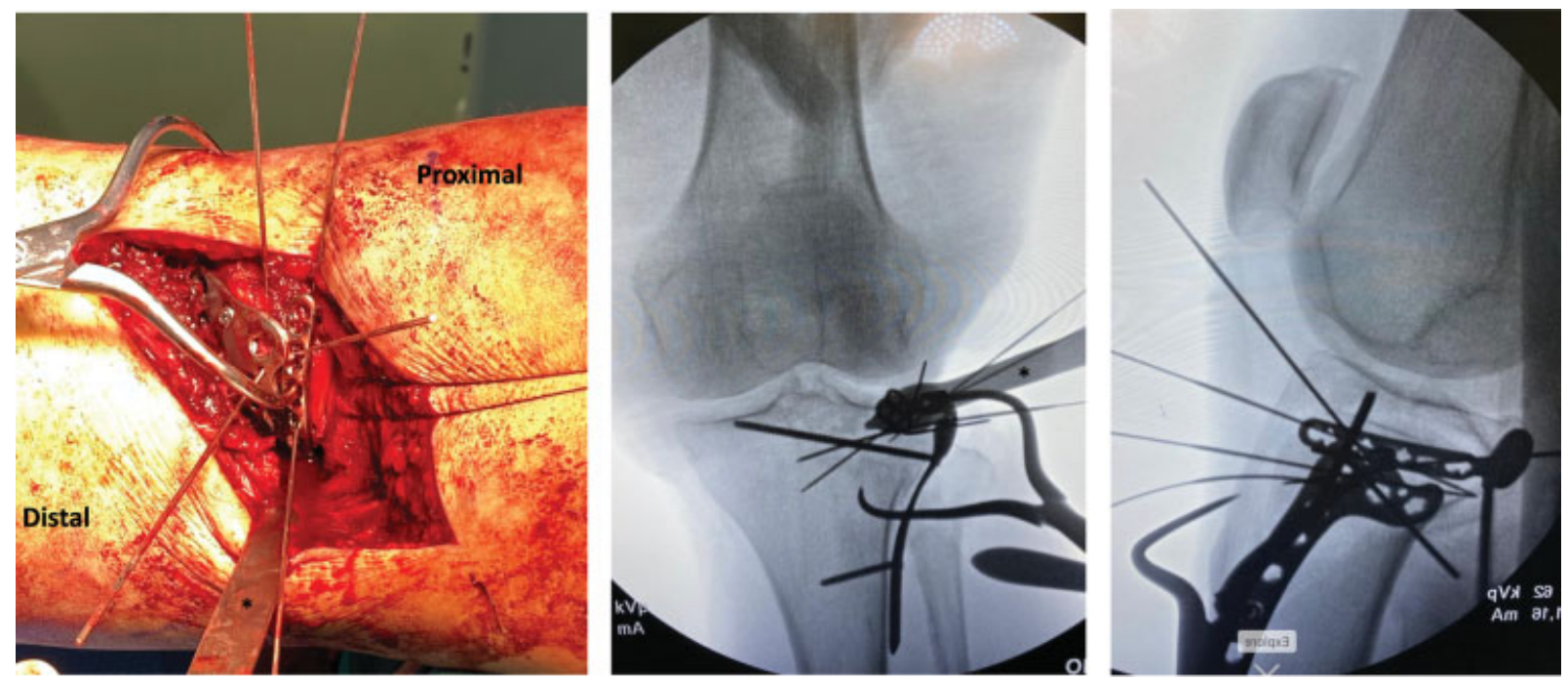

Fig. 5 Posicionamiento de placa rim a través del abordaje anterolateral extendido: se evidencia el uso de la paca rim. El segmento trebolado de la placa queda hacia posterior, entregando soporte al fragmento posterolateral (FPL). La pinza de reducción asiste para mantener la placa adosada al hueso a nivel posterior. Se observa como el retractor de Hohmann $\left({ }^{*}\right)$, ubicado en la región posterior de la tibia, permite una visualización directa del FPL para su reducción y una correcta posición de la placa.
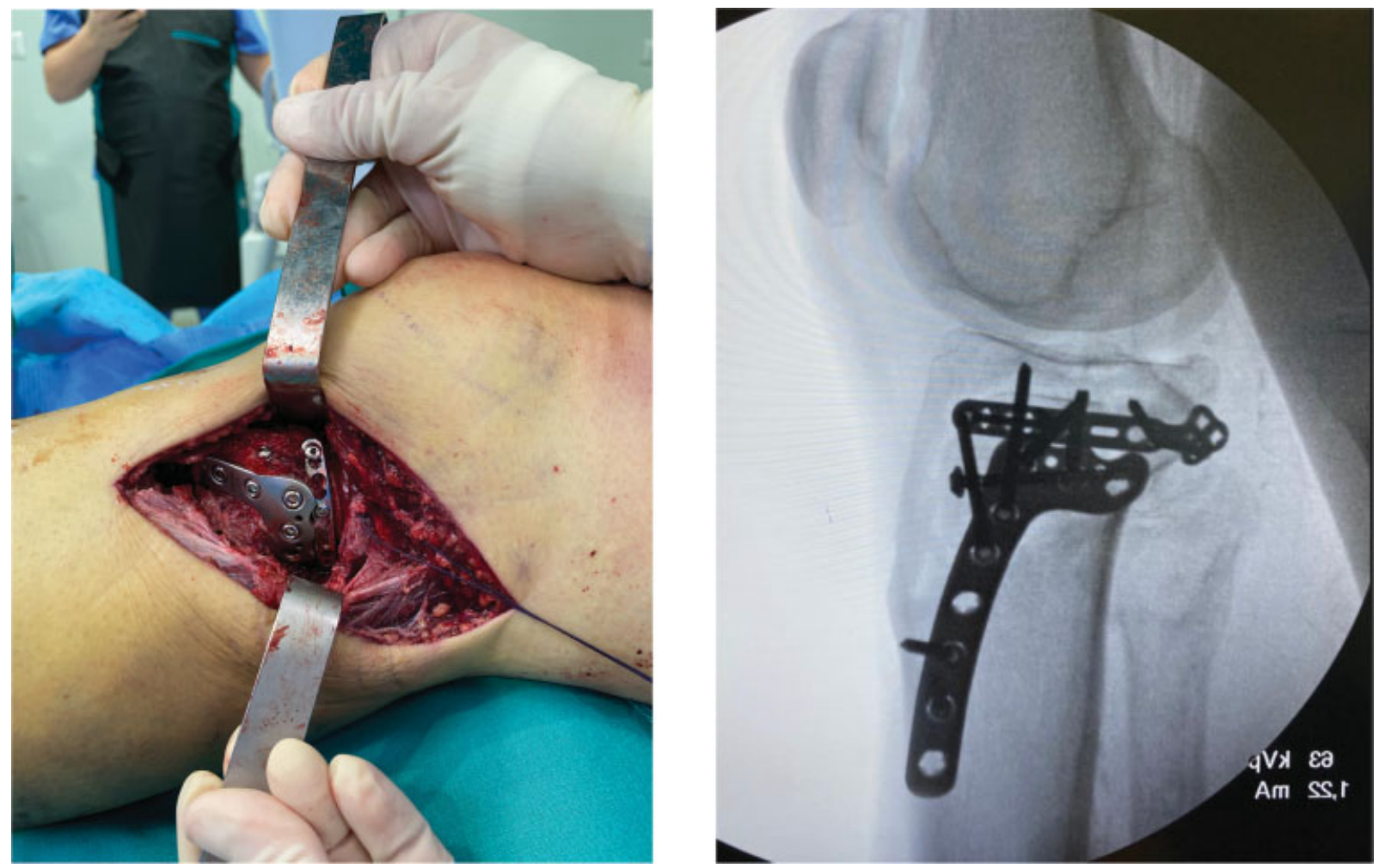

Fig. 6 Fractura reducida y estabilizada. Se observa el resultado final intraoperatorio. La fractura fue reducida y estabilizada por medio de una placa bloqueada de tibia proximal lateral, la placa rim, y un tornillo canulado en dirección anteroposterior.

\section{Discusión}

La detección de FPL en fracturas de platillos tibiales es más frecuente que lo que se creía. Mientras Partenheimer et al. ${ }^{18}$ describieron una tasa de $7 \%$, la literatura ${ }^{5-7}$ actual describe hasta un $80 \%$ de detección con una tasa de malreducción entre $50-70 \%$. Estos fragmentos son difíciles de reducir, principalmente por la obstaculización de la cabeza fibular y los componentes de la esquina posterolateral. ${ }^{19}$

La reducción anatómica y estabilización de las fracturas de platillo tibiales ha demostrado buenos resultados a largo plazo; la malreducción de los FPLs se ha asociado con inestabilidad de rodilla durante la flexión. ${ }^{17,19}$ Sin embargo, no existe consenso sobre cómo deben ser manejados estos FPLs. ${ }^{8,9}$ Cuando existen rasgos con separación (split) articular, es de preferencia utilizar 


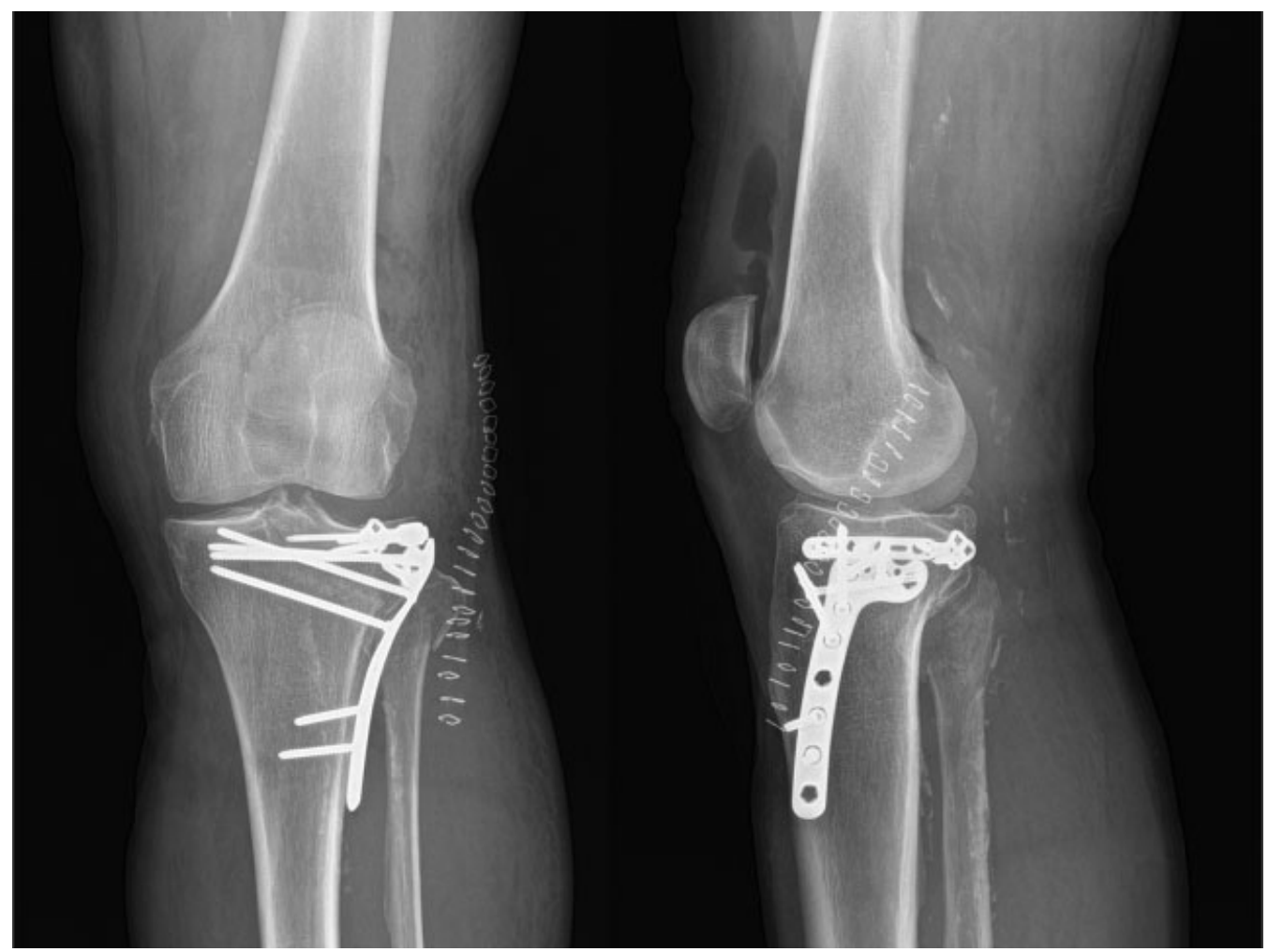

Fig. 7 Radiografías postoperatorias. Se observa una adecuada reducción de la superficie articular (escalón $<2$ mm) y del ancho del platillo tibial. La placa rim se encuentra ubicada en el margen proximal del platillo lateral, desde la región posterolateral hasta la región anterolateral.

placas de sostén (butress) para contrarrestar la fuerza cizallante de la carga axial. ${ }^{3,11,20,21}$ Idealmente, estas placas deben ser colocadas de forma paralela al rasgo y apoyarse en el ápex de la fractura. ${ }^{3,21}$ En casos en los que el ápex se encuentre posterior, variados abordajes han sido descritos para lograr una reducción y estabilización satisfactoria. ${ }^{19,21-23}$ Dentro de estos, existe una tendencia a preferir abordajes posteriores directos, en posición decúbito prono, especialmente cuando existe una fractura articular en el plano coronal con un ápex posterior. No obstante, en caso de existir un rasgo anterior a la línea media del platillo, requeriría reposicionar al paciente durante el procedimiento, aumentando el tiempo quirúrgico y, con ello, el riesgo de infección. ${ }^{16}$ Adicionalmente, existe riesgo de dañar estructuras neurovasculares, tales como la arteria tibial anterior, ${ }^{14}$ y también deben sumarse las consecuencias fisiológicas de mantener al paciente anestesiado en decúbito prono.
Por lo tanto, en caso de presencia de un FPL no cizallante, es importante evaluar si es realmente necesario realizar un abordaje posterior directo. El presente artículo ilustra un abordaje quirúrgico que permite operar al paciente en posición decúbito supino, y un método de estabilización óptimo para FPL conminutos y depresiones que requieran contención. Cho et al. ${ }^{13}$ fueron los primeros en describir este abordaje y método de fijación, comentando las siguientes ventajas: 1) exposición completa de las partes lateral y pósterolateral del platillo lateral, permitiendo espacio suficiente para evitar una osteotomía de la cabeza fibular; 2 ) posición del paciente en decúbito supino; 3 ) posibilidad de reducir y comprimir la fractura bajo visión directa; 4) fijación simultánea de las columnas anterolateral y posterolateral por medio de placas; 5) visión simultánea del FPL y la superficie articular, lo que no sería posible con el abordaje posterior directo; 6) puede ser ejecutado por cirujanos con menor

Caja 1 Consejos y errores de la técnica quirúrgica

\begin{tabular}{|c|c|}
\hline Consejos & Errores \\
\hline $\begin{array}{l}\text { 1. Al momento de disecar el espacio paraligamento colateral } \\
\text { lateral, flectar la rodilla en } 90^{\circ} \text { para relajar el ligamento y el } \\
\text { nervio peroneo común. } \\
\text { 2. Doblar la placa rim de forma parcelada (espacio tras espacio), y } \\
\text { asegurar que se doble la punta anterior para evitar prominen- } \\
\text { cias. } \\
\text { 3. Colocar una pinza de reducción con punta a través de la placa } \\
\text { rim, idealmente en uno de los orificios del trébol para asegurar } \\
\text { su adhesión a la cortical posterior. }\end{array}$ & $\begin{array}{l}\text { 1. Considerar que todos los fragmentos posterolaterales del } \\
\text { platillo tibial pueden ser reducidos correctamente desde un } \\
\text { abordaje anterolateral estándar, o a través de tornillos en } \\
\text { dirección ánteroposterior. } \\
\text { 2. Extender la disección del espacio paraligamento colateral } \\
\text { lateral proximal a la línea articular. Esto puede resultar en daño } \\
\text { al tendón poplíteo y generar inestabilidad iatrogénica. } \\
\text { 3. Colocar placa lateral de tibia proximal muy proximal (subcon- } \\
\text { dral), impidiendo el uso de la placa rim } \\
\text { 4. Doblar la placa rim con un material inadecuado, puede resultar en } \\
\text { deformación de los orificios e impedir el bloqueo de tornillos. }\end{array}$ \\
\hline
\end{tabular}


experiencia, disminuyendo la potencial limitación de los abordajes posteriores directos; 7) el retiro de material de osteosíntesis es más sencillo y menos riesgoso; y 8) no requiere identificar el nervio peroneo común ni estructuras vasculares posterolaterales. Además, los autore ${ }^{13} \mathrm{~s}$ describen que es una opción segura, sin complicaciones ligamentarias ni neurovasculares reportadas.

Es importante mencionar que la placa rim no funciona como sostén, por lo que los autores de este estudio consideran que el abordaje y método de reducción descritos serían insuficientes para rasgos cizallantes en plano coronal con ápex en la región posterolateral. Los consejos y errores de la técnica quirúrgica se describen en la Caja 1.

\section{Conclusión}

El abordaje anterolateral extendido a través del espacio paraLCL y la fijación con placa rim es una técnica quirúrgica óptima para la visualización, reducción y fijación de FPLs conminutos y depresiones articulares que requieran contención.

\section{Conflicto de intereses}

Dr. F. Figueroa relata que es miembro editor de la Acta Ortopedica Mexicana. Los otros autores declaron no haber conflicto de intereses.

\section{Agradecimientos}

Quisiéramos agradecer a Karen Illesca, técnica en enfermería e instrumentación quirúrgica, por ser parte esencial del equipo quirúrgico, y a Arielle Ergas, por la ilustración aportada.

\section{Referencias}

1 Luo CF, Sun H, Zhang B, Zeng BF. Three-column fixation for complex tibial plateau fractures. J Orthop Trauma 2010;24(11): 683-692. Doi: 10.1097/BOT.0b013e3181d436f3

2 Rossmann M, Fensky F, Ozga AK, et al. Tibial plateau fracture: does fracture classification influence the choice of surgical approach? A retrospective multicenter analysis. Eur J Trauma Emerg Surg 2020;(0123456789):1-7. Doi: 10.1007/s00068-020-01388-Z

3 Kfuri M, Schatzker J. Revisiting the Schatzker classification of tibial plateau fractures. Injury 2018;49(12):2252-2263. Doi: 10.1016/j.injury.2018.11.010

4 Meinberg EG, Agel J, Roberts CS, Karam MD, Kellam JF. Fracture and Dislocation Classification Compendium-2018. J Orthop Trauma 2018;32(9781461479864, Suppl 1)S1-S170. Doi: 10.1097/BOT.0000000000001063

5 Zhai Q, Luo C, Zhu Y, et al. Morphological characteristics of splitdepression fractures of the lateral tibial plateau (Schatzker type II): a computer-tomography-based study. Int Orthop 2013;37 (05):911-917. Doi: 10.1007/s00264-013-1825-5

6 Adams JD Jr, Della Rocca GJ. Management of Posterior Articular Depression in Tibial Plateau Fractures. J Knee Surg 2016;29(01): 28-33. Doi: 10.1055/s-0035-1566737

7 Meulenkamp B, Martin R, Desy NM, et al. Incidence, Risk Factors, and Location of Articular Malreductions of the Tibial Plateau. J
Orthop Trauma 2017;31(03):146-150. Doi: 10.1097/BOT.000000 0000000735

8 Warner BT, Moulton SG, Cram TR, LaPrade RF. Anatomic Reconstruction of the Proximal Tibiofibular Joint. Arthrosc Tech 2016;5(01):e207-e210. Doi: 10.1016/j.eats.2015.11.004

9 Yi Z, Hui S, Binbin Z, et al. A new strategy to fix posterolateral depression in tibial plateau fractures: Introduction of a new modified Frosch approach and a "Barrel hoop plate" technique. Injury 2020;51(03):723-734. Doi: 10.1016/j.injury.2020.01.023

10 Solomon LB, Stevenson AW, Lee YC, Baird RPV, Howie DW. Posterolateral and anterolateral approaches to unicondylar posterolateral tibial plateau fractures: a comparative study. Injury 2013;44(11):1561-1568. Doi: 10.1016/j.injury.2013.04.024

11 Zhang W, Luo CF, Putnis S, Sun H, Zeng ZM, Zeng BF. Biomechanical analysis of four different fixations for the posterolateral shearing tibial plateau fracture. Knee 2012;19 (02):94-98. Doi: 10.1016/j.knee.2011.02.004

12 Hangody L, Dobos J, Baló E, Pánics G, Hangody LR, Berkes I. Clinical experiences with autologous osteochondral mosaicplasty in an athletic population: a 17-year prospective multicenter study. Am J Sports Med 2010;38(06):1125-1133

13 ChoJW, Samal P, Jeon YS, Oh CW, Oh JK. Rim Plating of Posterolateral Fracture Fragments (PLFs) Through a Modified Anterolateral Approach in Tibial Plateau Fractures. J Orthop Trauma 2016;30 (11):e362-e368. Doi: 10.1097/BOT.000000000 0000638

14 Heidari N, Lidder S, Grechenig W, Tesch NP, Weinberg AM. The risk of injury to the anterior tibial artery in the posterolateral approach to the tibia plateau: a cadaver study. J Orthop Trauma 2013;27(04):221-225. Doi: 10.1097/BOT.0b013e318271f8f0

15 Chen WT, Zhang YQ Chang SM. Posterolateral approach for plating of tibial plateau fractures and the risk of injury to the anterior tibial vessels. J Orthop Trauma 2013;27(09):e228-e229. Doi: 10.1097/BOT.0b013e31829ff3e9

16 Colman M, Wright A, Gruen G, Siska P, Pape HC, Tarkin I. Prolonged operative time increases infection rate in tibial plateau fractures. Injury 2013;44(02):249-252. Doi: 10.1016/j.injury.2012.10.032

17 Cho JW, Kim J, Cho WT, et al. Approaches and fixation of the posterolateral fracture fragment in tibial plateau fractures: a review with an emphasis on rim plating via modified anterolateral approach. Int Orthop 2017;41(09):1887-1897. Doi: 10.1007/s00264-017-3563-6

18 Partenheimer A, Gösling T, Müller M, et al. [Management of bicondylar fractures of the tibial plateau with unilateral fixedangle plate fixation]. Unfallchirurg 2007;110(08):675-683. Doi: 10.1007/s00113-007-1271-1

19 Frosch KH, Balcarek P, Walde T, Stürmer KM. A new posterolateral approach without fibula osteotomy for the treatment of tibial plateau fractures. J Orthop Trauma 2010;24(08):515-520. Doi: 10.1097/BOT.0b013e3181e5e17d

20 Wang Y, Luo C, Zhu Y, et al. Updated Three-Column Concept in Surgical Treatment for Tibial Plateau Fractures - A Prospective Cohort Study of 287 Patients. Vol 47;Elsevier Ltd; 2016. Doi: 10.1016/j.injury.2016.04.026

21 Kfuri M, Schatzker J, Castiglia MT, Giordano V, Fogagnolo F, Stannard JP. Extended Anterolateral Approach for Complex Lateral Tibial Plateau Fractures. J Knee Surg 2017;30(03): 204-211. Doi: 10.1055/s-0037-1598077

22 Carlson DA. Posterior bicondylar tibial plateau fractures. J Orthop Trauma 2005;19(02):73-78. Doi: 10.1097/00005131-20050200 0-00001

23 Garner MR, Warner SJ, Lorich DG. Surgical Approaches to Posterolateral Tibial Plateau Fractures. J Knee Surg 2016;29 (01):12-20 